\section{GRAVITATION}

\section{Radiation and Collapse}

\section{from a Correspondent}

Symposium 64 of the Extraordinary General Assembly of the International Astronomical Union, held in Warsaw on September 5-8 to commemorate the 400th anniversary of Nicholas Copernicus, reflected the growing interest in general relativity among astronomers and astrophysicists. Entitled "Gravitational Radiation and Gravitational Collapse" the programme ranged over most of the areas where it is believed that general relativistic effects are or soon will be susceptible of observation.

Pre-eminent among these areas is the question of the claims of $J$. Weber (University of Maryland) to have observed gravitational radiation. His colleague C. Misner reviewed the largely unsuccessful attempts of theoreticians to create models of the copious sources of gravitational waves required by Weber's claims. A short report by A. Poveda (University of Mexico) of a new (remarkably low) upper limit to the rate at which the Galaxy is losing mass of 1 solar mass per year, deduced from the orbits of globular clusters, will not make this task any easier. A. Tyson (Bell Laboratories) followed with an account of the current observational situation in which several groups have sought to repeat Weber's experiments with detectors which are claimed to be of equal or better sensitivity than Weber's 196970 antennae and have been unsuccessful.

An afternoon session was devoted to a discussion of these results between the parties involved. In particular $\mathbf{R}$. Drever (University of Glasgow), P. Kafka (Max Planck Institute, Munich) and Tyson presented results which indicate that either Weber has not been seeing gravitational waves or that the source has weakened since 1970 . On the other hand Weber made renewed claims concerning coincidences both between his own antennae and between his and those of D. Douglas (University of Rochester). The latter, which amount to some fifty-four zero delay coincidences above random in 7 days worth of data, represent an effect at the 2.7 standard deviation level. The fact that Douglas's instrument operates at $710 \mathrm{~Hz}$ would indicate that if this effect is real the signals really are broad band, as has long been suspected. It is hoped that the resolution of the puzzle will be brought about by further plans to exchange tapes and subject the raw data to a variety of signal processes, each favoured by one or other experimental groups. Of future plans perhaps the most striking was V. Braginsky's (State University of Moscow) idea of using a massive monocrystal instead of an aluminium bar with a resultant very high quality factor to look for the sort of signals astronomers expect from nearby galaxies or gravitational bremsstrahlung produced by close encounters of stars-an idea emphasised by Ya. Zeldovich (Institute of Applied Mathematics, Moscow). Some encouraging progress was also reported on the supercooled detectors.

In the light of this it was amusing to hear J. Faulkner (Lick Observatory, California) suggesting that the effects of gravitational radiation might be found on our own doorstep, so to speak, in that mass transfer taking place in very short period binary star systems seems to be controlled in part by the radiation of angular momentum by gravitational waves.

Rather more positive were the sessions on collapse and black holes. R. Giacconi (American Science and Engineering) summarised the wealth of data from optical, radio and most importantly X-ray observation indicating that $\mathrm{Cyg}$ X-1 and SMC X-1 may possibly contain black holes. R. Kraft (Lick Observatory) was able considerably to strengthen the evidence by obtaining a lower bound to the distance to Cyg X-1 and hence a lower bound of $6 M_{\odot}$ to the mass of the unseen secondary. There was, of course, much discussion of accretion models for such X-ray sources and this underlined the importance of numerical work by S. Teukolsky and W. Press (California Institute of Technology) on the stability of rotating black holes and although not everybody will agree with A. Starobinsky (Landau Institute, Moscow) that his ingenious analytical results on superradiant scattering of waves off black holes, thereby increasing the energies of the waves at the expense of the black hole, guarantees that stability, they clearly represent important advances in black hole theory. The larger issue of "naked singularities" and whether a black hole can shed the respectable clothing of its event horizon to reveal the singularities inside in more extreme situations than those envisaged in the perturbation approach of Press, Teukolsky and Starobinsky were taken up by R. Penrose (University of Oxford) who provided a useful new definition of naked singularities and repeated previous warnings that the mathematical evidence against such objects is largely non-existent.

Among other topics discussed was the report by B. Carr (University of Cam-

\title{
Markers of CNS Malformation
}

SPINA bifida and anencephaly, two closely related malformations of the central nervous system (CNS), are comparatively common congenital disorders in the population of the United Kingdom, with a combined incidence of about 4 in every 1,000 births. Anencephalics die at birth, but after surgery, children with spina bifida live for a number of years with a high degree of physical and mental handicap. The policy of radical surgical intervention, which prolongs the lives of grossly incapacitated children and puts an enormous burden on their families, has recently been called in question.

A little over a year ago Brock and his colleagues (Lancet, ii, 197, 1252; 1972) reported that concentrations of $\alpha$ foetoprotein (AFP) were higher in the amniotic fluids of pregnancies leading to anencephaly and spina bifida. Their study was retrospective---that is, made on amniotic fluids obtained for other reasons and where the outcome of the pregnancy was already knownbut it raised the hope of early diagnosis of these conditions followed by termination of the pregnancy. These hopes have now been realised in prospective studies (Lorber et al., Lancet, i, 1187; 1973; Allan et al., Lancet, ii, 522; 1973) and it is becoming an aspect of routine medical care to monitor pregnancies at risk for CNS malformations through AFP levels in the amniotic fluid. The report of Macri et al. in Nature New
Biology next Wednesday (November 21) adds a new dimension to these studies in that they have demonstrated a CSFspecific protein in the amniotic fluid of foetal Lewis rats with incomplete neural tube closures. Similar proteins are known in human CSF and, if they leak into the amniotic fluid, may provide another parameter to add to AFP in the early diagnosis of CNS malformations.

The real problem, however, in the antenatal diagnosis of spina bifida is identifying the at-risk pregnancy. Ascertainment through the previous birth of an affected child will allow detection of only a small number of cases, since more than $90 \%$ of such children will be born to mothers who have no relevant medical history to alert the obstetrician. Monitoring all pregnancies through early amniocentesis and protein analysis is clearly out of the question. A start to the problem has been made by Brock et al. (Lancet, ii, 923; 1973) who diagnosed an anencephalic pregnancy through AFP levels in the mother's blood. Presumably this foetal protein crossed the placental barrier from the amniotic fluid. The same is likely to occur with spina bifida, because amniotic fluid AFP is every bit as high. But before large-scale monitoring of maternal serum is contemplated it would be as well to have other markers of CNS malformation. The CSF-specific protein described by Macri et al. may be just such a protein. 